$i$ erfc $z$ and $i^{2}$ erfc $z$ correct to 6 significant figures ( 7 s.f. for $z>1$ ) using single precision on a computer with word length of 8 decimal places, for all $z$ for which $e^{-z^{2}}$ can be calculated correctly. To obtain greater accuracy, it is necessary either to use double precision or to use more than two different expansions for each function. From Gautschi's formula [2] for the number of terms required for calculation by backward recurrence, we see that that method will be better (for 7 s.f. accuracy) if all the $z$ 's of interest are greater than about 2.5. The advantage accruing from the use of Chebyshev approximations would be still greater for multiple-precision calculations of very high accuracy.

Battelle Memorial Institute

Columbus, Ohio

1. M. Abramowitz \& I. A. Stegun, (Editors), Handbook of Mathematical Functions, with Formulas, Graphs, and Mathematical Tables, National Bureau of Standards Applied Mathematics Series, No. 55, U. S. Government Printing Office, Washington, D. C., 1964; reprint, 1965. MR 29 \#4914; MR 31 \#1400.

2. W. GaUtSCHI, "Recursive computation of the repeated integrals of the error function," Math. Comp., v. 15, 1961, pp. 227-232. MR 24 \#B2113.

3. W. R. WILCOX "Asymptotic computation of the repeated integrals of the error function complement," Math. Comp., v. 18, 1964, pp. 98-105. MR $28 \# 1327$.

4. C. W. Clenshaw, "The numerical solution of linear differential equations in Chebyshev series," Proc. Cambridge Philos. Soc., v. 53, 1957, pp. 134-149. MR 18, 516.

5. J. L. FIELDS \& J. WIMP, "Expansions of hypergeometric functions in hypergeometric functions," Math. Comp., v. 15, 1961, pp. 390-395. MR 23 \#A3289.

6. J. WIMP \& Y. L. LUKE, "Expansion formulas for generalized hypergeometric functions," Rend. Circ. Mat. Palermo, 2, v. 11, 1962, pp. 351-366. MR 29 \#3681.

7. Y. L. LUKE \& J. WIMP, "Jacobi polynomial expansions of a generalized hypergeometric function over a semi-infinite ray," Math. Comp., v. 17, 1963, pp. 395-404. MR 28 \#255.

\title{
An Integral Representation for the Modified Bessel Function of the Third Kind, Computable for Large, Imaginary Order
}

\section{By James D. Lear and James E. Sturm}

The one-dimensional Schroedinger equation describing the quantum-mechanical motion of a particle of total energy $E$ and mass $\mu$ in a potential field of the form:

$$
\begin{array}{ll}
V=B \exp (-r / a) & \text { for } r>0 \\
V=\infty & \text { for } r \leq 0
\end{array}
$$

has, as time-independent solutions, the functions

$$
\left(\frac{\nu \sinh \pi \nu}{\pi}\right)^{1 / 2} K_{i \nu}(z)
$$

where $\nu=2 a\left(2 \mu E / \hbar^{2}\right)^{1 / 2}, z=2 a B e^{-r / 2 a}, K_{i \nu}(z)$ is the modified Bessel function of the third kind, and the normalization is to unit amplitude of the asymptotic ( $r$ increasing) solution [1]. In attempting to compute values for $K_{i \nu}(z)$ through use of the representation: 


$$
K_{i \nu}(z)=\int_{0}^{\infty} \exp [-z \cosh \phi] \cos \nu \phi d \phi
$$

evaluated by Simpson's rule on a digital computer, we found that computational precision was quickly lost for $\nu>15$ and $\nu / z$ around or exceeding unity. The reason for this is evident when one considers the fact that, while $K_{i \nu}(z)$ has a maximum amplitude of the order $(\pi / \nu \sinh \pi \nu)^{1 / 2}$, the maximum amplitude of the integrand in (1) is independent of $\nu$. Hence, as $\nu$ increases, the accuracy requirement for the integration increases and eventually exceeds that afforded by the word sizes of most electronic computing machines. Below is given a simple generalization of (1) by which the excessive accuracy requirement can be replaced by an increase in the time required for the numerical integration.

We note that $K_{i v}(z)$ is a real function related to the Hankel function of the first kind via:

$$
K_{i \nu}(z)=\frac{\pi}{2} \exp [\pi i(1+i \nu) / 2] H_{i \nu}^{(1)}(i z) .
$$

Beginning with the integral representation for the Hankel function:

$$
H_{i \nu}^{(1)}(i z)=\frac{1}{\pi} \int_{\text {path }} \exp [-z \cos \phi-\nu(\phi-\pi / 2)] d \phi
$$

where the path in the complex $\phi$ plane extends from $i \infty$ to $-i \infty$ within the strip $|\operatorname{Re} \phi|<\pi / 2$, we let $w=u+i v=-i \phi$, reverse the $w$ plane path, and multiply the resulting expression by $-(\pi / 2) \exp [\pi i(1+i \nu) / 2]$ to obtain, considering $(2)$ :

$$
K_{i \nu}(z)=\frac{1}{2} \int_{-\infty+i b}^{\infty+i a} \exp [-z \cosh w-i \nu w] d w, \quad|a|,|b|<\pi / 2 .
$$

With $w=u+i v,(4)$ can be expressed as:

$$
K_{i \nu}(z)=\frac{1}{2} \int_{-\infty+i b}^{\infty+i a} e^{\nu v} \exp [-z \cosh u \cos v] \cos (z \sinh u \sin v+\nu u) d w
$$

where we have used the fact that $K_{i \nu}(z)$ is real to eliminate the imaginary part of (4). If we now let $v$ be constant along the integration path, we obtain:

$$
K_{i \nu}(z)=\int_{0}^{\infty} e^{\nu v} e^{-z \cosh u \cos v} \cos (z \sinh u \sin v+\nu u) d u
$$

which, for $v=0$, reduces to the standard definition.

The advantage of (6) comes in the dependence of the integrand amplitude on $v$. By adjusting $v$ to be a negative constant (greater than $-\pi / 2$ ), the large fluctuations of the integrand can be suppressed to tolerable magnitudes. The slower convergence of (6), while not as serious a problem as the former requirement of huge word sizes, will limit the applicability of the representation to a range of $\nu$ within which error accumulation in the integration can be held within tolerable limits.

The table given below shows how the accuracy of an evaluation depends on the choice of $v$ for constant $( \pm 1 \%)$ values of $\cos v \cosh B . B$ is the upper limit on $u$ in the integration and $D$ is the size of the Simpson's rule increment in $u$, both parameters being chosen to give a 6-significant-figure accuracy limit for $v$ approaching $-\pi / 2$. 


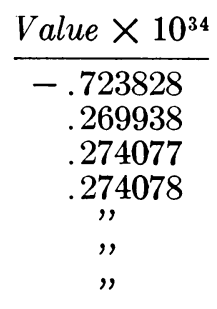

\begin{tabular}{ccc}
\multicolumn{3}{c}{ Evaluation of $K_{i 50}(20)$} \\
\hline$v$ & $\frac{B}{.0025}$ \\
-1.00 & 3.65 &,$"$ \\
-1.20 & 4.00 & $"$, \\
-1.40 & 4.80 & .005 \\
-1.58 & 5.45 & $"$, \\
-1.52 & 5.60 &
\end{tabular}

Acknowledgement. This work was supported by the Atomic Energy Commission under Contract No. A'T(30-1)-2772 which included access to the CDC-6600 facility at the Courant Institute for Mathematical Sciences at New York University.

Lehigh University

Bethlehem, Pennsylvania

E. I. D॥ Pont Nemours

Wilmington, Delaware 19898

1. J. M. JACKson \& N. F. Motr, "Energy exchange between inert gas atoms and a solid surface," Proc. Roy. Soc. London Ser. A, v. 137, 1932, pp. 703-717.

\title{
Mixed Algebraic-Exponential Interpolation Using Finite Differences
}

\author{
By J. W. Layman
}

The use of finite differences in exponential polynomial interpolation was introduced in [1], where an algorithm was developed which triangularizes the system of cquations that determines the coefficients in the interpolating exponential polynomial. In the present note we show that a similar finite-difference algorithm also exists for interpolation by a mixed algebraic-exponential polynomial of the form

$$
P(x)=\sum_{n=1}^{N} \sum_{m=0}^{m_{n}} a_{n m} x^{(m)} n^{x}
$$

for $x=0,1,2, \cdots, \sum_{n=1}^{I}\left(m_{n}+1\right)-1$. The symbol $x^{(m)}$ represents the factorial power function $x(x-1) \cdots(x-m+1)$.

We require the basic difference operations $E$ and $\Delta$ and, in addition, the diagonal difference $S$ defined by $S f(x)=\Delta^{x} f(0)$. The diagonal difference is more precisely defined in [1] and certain difficulties in interpretation are resolved there. These arise when taking higher-order diagonal differences by iteration, $S^{n} f(x)=S S^{n-1} f(x)$.

The following properties and formulas involving the diagonal-difference opera-

Received April 28, 1966. Revised December 5, 1966. 